\title{
Experiment-simulation Study on Noise Reduction of Cylinder Shell with Horn Helmholtz Resonator
}

\author{
Wenjie YAN, Shilin YAN, Bin LI, Yongjing LI
}

\begin{abstract}
This paper focuses on the noise reduction of the cylindrical structure at low frequency $(130 \mathrm{~Hz}-180 \mathrm{~Hz})$. The low-frequency noise response spectrum in the cylindrical cavity is obtained by using the Helmholtz resonator $(H R)$ to reduce the noise peak amplitude. The acoustic simulation software Virtual.Lab is used to establish the finite element model of the cylindrical shell with HR, and to obtain the low-frequency acoustic response in the cylindrical cavity. The simulation model is validated by the experimental results. Then, the influence of installation position, the number of installed resonators and the resonators with different resonance frequencies in the cylindrical cavity are discussed. The results indicate that both the noise reduction band and peak amplitude are increased by installing the HR's on the cylinder shell. The noise reduction of the cylinder shell with HR installed on the upper position is larger than other situations. As the number of resonators increased, the frequency range of the noise reduction in the cylindrical cavity gradually increases, and the noise reduction of the cylinder cavity increases first and then decreases.
\end{abstract}

Keywords: cylindrical shell; Helmholtz resonator; low-frequency noise; noise reduction

\section{INTRODUCTION}

The launch vehicle is mainly affected by jet noise [1] and aerodynamic noise during flight [2], and the sound pressure level outside the cavity during the launch process can reach more than $170 \mathrm{~dB}$ [3]. The external noise of the rocket fairing which poses a threat to the safety of the internal payload is transmitted to the inside of the fairing through transmission and radiation in a wide frequency and random form. Therefore, reducing the noise in the fairing cavity is very important to protecting the payload and improving the level of the launch vehicle.

The cylindrical shell, which is a part of the rocket fairing, can be approximated as a cylindrical shell structure. Optimizing the structure of the cylindrical shell is an effective way to reduce vibration and noise. However, there is still a noise peak in the cylindrical cavity at the lowfrequency range by optimizing the cylinder structure [4]. The European Aviation Administration and Japan have proposed to use a method of laying sound-absorbing materials (melamine foam board, glass fiber acoustic blanket, polyamide foam, etc.) on the inner wall of a cylindrical shell to reduce the noise $[5,6]$. The method of adding absorbing material has better noise reduction capability at high frequency. So, the acoustic response in the cylindrical cavity at the middle and low-frequency range still needs to be reduced.

The HR is a kind of widely used acoustic device that typically has a closed cavity and a small neck opening. The HR has a strong sound absorption capacity at its resonant frequency (generally, the frequency is low). The experimental results of Tang et al. [7] show that a tapered neck can improve the sound absorption performance of the HR. Liu Haitao et al. [8] corrected the acoustic length of the air in the conical neck and obtained a modified onedimensional model of the conical neck HR's transmission loss. Borchers et al. [9] designed a noise reduction device with a wide-band noise absorption performance, and the resonance frequency calculation formulas of the curved neck HR using a one-dimensional lumped parameter model are given. However, the design of the resonator is a difficult work for the reason that the modified parameters need to be obtained by experimental methods.

In this paper, the HR with 3 kinds of neck types are compared by using the lumped parameter method. The noise reduction test platform which has been built by our team was used to test the noise reduction of the cylindrical shell with HR. The simulation model was established by the finite element method. Then, the influence of the HR's position and number in the cylindrical shell to the noise reduction in the cylindrical cavity are discussed.

\section{THE HORN HELMHOLTZ RESONATOR}

The HR mainly absorbs noise whose frequency is close to its resonant frequency. The reason for HR achieving the purpose of absorbing noise is that part of the dynamic energy is dissipated into internal energy in the course of external noise propagation into the cavity of the resonator through the neck. The schematic diagram of the structure of the horn HR and coordinate system is shown in Fig. 1. The HR consists of a cylinder, a bottom circular plate, a neck curved surface, and a transition surface between the neck and the cylinder, where $l$ is the length of the neck, $a_{l}$ is the throat radius, $a_{0}$ is the resonator opening radius, $a$ is the radius of the bottom, $L$ is the side cylinder height, $V$ is the acoustic cavity of the resonator, and $r_{c}$ is the radius between the neck and the top opening surface.

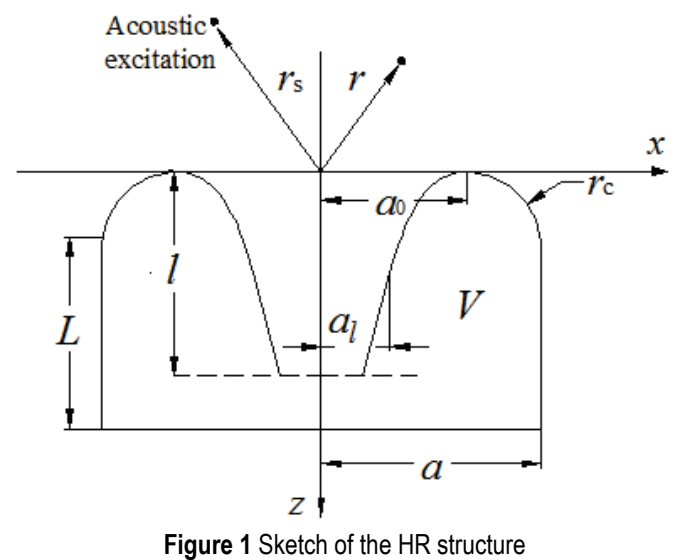

It is assumed that the HR is located on the infinite baffle plate, there is a point sound source in the space $r_{s}$. Under the low-frequency approximation, the air vibration radiation field of the HR is regarded as the sound field of infinite rigid piston radiation. The Green's function method 
is used to obtain the radiated acoustic impedance at the resonator opening.

$z_{p 0} \approx \frac{\rho_{0} \omega^{2} \pi a_{0}^{2}}{2 \pi c_{0}}-i \rho_{0} \omega \frac{8 a_{0}}{3 \pi}$

where $\rho_{0}$ is the air density, $\omega$ is the angular frequency, and $c_{0}$ is the speed of sound in the air. The sound pressure of HR throat $(z=1)$ is approximated as two parts: (1) The sound pressure caused by the change of cavity pressure inside the resonator. (2) If $a<<V^{1 / 3}$, the sound pressure radiated by the piston can be approximated by the sound pressure of the HR neck air vibrating in the throat [10] assuming that the piston vibration is positive toward the external radiation. Therefore, the acoustic radiation impedance at the resonator opening is

$z \approx \frac{\rho \omega \pi a}{2 \pi c}-i \rho \omega \varsigma a$

where $\varsigma$ is the coefficient related to the shape of the section. When the neck is deeply extended into the cavity, it is generally approximated by 0.61 [11]

It is assumed that the air vibration phase in the neck of the HR is the same, and the vibration velocity of the air particles in the same plane $\bar{v}_{z}$ is equal. Obviously, the vibration of the neck air is caused by the pressure difference between the two ends $\Delta p$, which is

$\Delta p=\rho_{0} l(-i \omega) \bar{v}_{z}(z, \omega)$

The specific acoustic impedance of the $\mathrm{HR} Z_{\mathrm{H}}$ can be obtained by substituting Eq. (1) and Eq. (2) into Eq. (3)

$$
Z_{\mathrm{H}}=z_{p o}+z_{p l} \frac{S_{0}}{S_{l}}-i\left(\rho_{0} \omega S_{0} \int_{0}^{l} \frac{1}{S(z)} \mathrm{d} z-\frac{\rho_{0} c_{0}^{2}}{\omega V} S_{0}\right)
$$

where, $S(z)$ is the cross-sectional area of the resonator neck at any position.
The resonance frequency of the exponential resonator $\left(f_{r}\right)$ satisfied the function $\operatorname{Im}\left(Z_{\mathrm{H}}\right)=0$. It could be expressed as follows

$$
f_{r}=\frac{c_{0}}{2 \pi} \sqrt{\frac{S_{0}}{V\left(l^{*}+\delta^{*}\right)}}
$$

where, $l^{*}$ and $\delta^{*}$ denote the effective length and neck correction of the air in the resonator neck respectively.

The reflection coefficient has the form [12]

$$
r=\left[\operatorname{Re}\left(Z_{\mathrm{H}}\right)-1\right] /\left[\operatorname{Re}\left(Z_{\mathrm{H}}\right)+1\right]
$$

The sound absorption coefficient of the $\operatorname{HR} \alpha$ is

$$
\alpha=1-|r|^{2}
$$

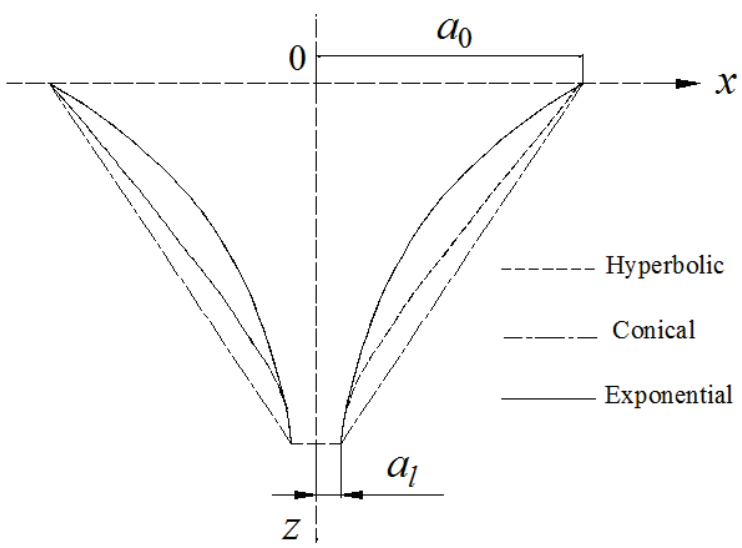

Figure 2 Sketch of different neck sections

As shown in Fig. 2, the neck section of the horn-shaped HR mainly includes exponential, hyperbolic and conical type. In the case of the structural appearance and structure of the HR having the same size, the neck effective length and correction calculation formulas of the three different

\begin{tabular}{|c|c|c|c|}
\hline Type & Exponential & Conical & Hyperbolic \\
\hline Sectional area $S_{z}$ & $S_{z}=S_{0} e^{-\delta z}$ & $S_{z}=S_{0}\left(1-\frac{\tau-1}{\tau} \chi\right)^{2}$ & $S_{z}=\frac{S_{0}}{\tau^{2}}\left[1+(\chi-1)^{2}\left(\tau^{2}-1\right)\right]$ \\
\hline Effective length $l^{*}$ & $l^{*}=\frac{\left(\mathrm{e}^{\delta l}-1\right)}{\delta}$ & $l^{*}=\tau l$ & $l^{*}=\frac{\tau^{2} \arctan \sqrt{\tau^{2}-1}}{\sqrt{\tau^{2}-1}} l$ \\
\hline Neck correction $\delta$ & $\delta^{*}=\frac{8 a_{0}}{3 \pi}+\varsigma a_{l}$ & $\delta^{*}=\frac{8 a_{0}}{3 \pi}+\varsigma a_{l}$ & $\delta^{*}=\frac{8 a_{0}}{3 \pi}+\varsigma a_{l}$ \\
\hline
\end{tabular}
types of sections can be obtained by the formulas (1), (2), (3), and (4) shown in Tab. 1.

Table 1 Several section types of neck calculation formula

where, $\delta$ is the meandering index of the exponential curve, $\tau$ is a dimensionless quantity $a_{0} / a_{l}$ and $\chi$ is a dimensionless quantity $z / l$. From Tab. 1, it can be known that, if the opening radius $a_{0}$ and throat radius $a_{l}$ are the same, the HR with different neck shapes have the same neck correction $\delta^{*}$, while the effective length is different in large $l^{*}$. Let the subscripts $e, c, h$ represent the exponential neck, the conical neck and the hyperbolic neck resonator respectively. When the basic dimensions of the resonator structure are the same, the effective length relationship between the HR's with different neck type is

$$
\left\{\begin{array}{lr}
l_{h}^{*}>l_{e}^{*}>l_{c}^{*} & 1<\tau<4.1 \\
l_{e}^{*}>l_{h}^{*}>l_{c}^{*} & \tau>4.1
\end{array}\right.
$$

For the HR with a cylindrical neck, the opening radius of the horn is generally much larger than the radius of the 
throat. The dimensionless quantity $\tau$ is easy to reach greater than 4.1 , so it is generally considered that $l_{e}^{*}>l_{h}^{*}>l_{c}^{*}$. By substituting the Eq. (8) to Eq. (5), it easily gets that the relationship between the resonant frequencies of the three types of HR's is $f_{e}>f_{h}>f_{c}>$ at the condition of $\tau>4.1$.

The exponential neck resonator has the largest effective length of the neck and the smallest resonance frequency under the condition that the resonator has a similar appearance and the same size. In practical applications, resonator volume can not be greatly changed due to the objective conditions, while the resonance frequency of the resonator, which has the exponential neck, can be easily changed by slightly changing the neck size. Therefore, HR with an exponential neck is applied to reduce the noise in the cylindrical cavity.

\section{EXPERIMENT}

\subsection{The Sound Absorption Coefficient of HR}

According to the formula of the resonance frequency and sound absorption coefficient of the exponential HR, an exponential neck HR whose resonance frequency is $154 \mathrm{~Hz}$ was designed and processed. As shown in Fig. 3a, the basic structural parameters of the resonator are total height $L=$ $83 \mathrm{~mm}$, neck length $l=70 \mathrm{~mm}$, neck opening diameter $D$ $=98 \mathrm{~mm}$, neck outer opening diameter $d=80 \mathrm{~mm}$, throat diameter $3.767 \mathrm{~mm}$, and the thickness of the resonator is 1 $\mathrm{mm}$.

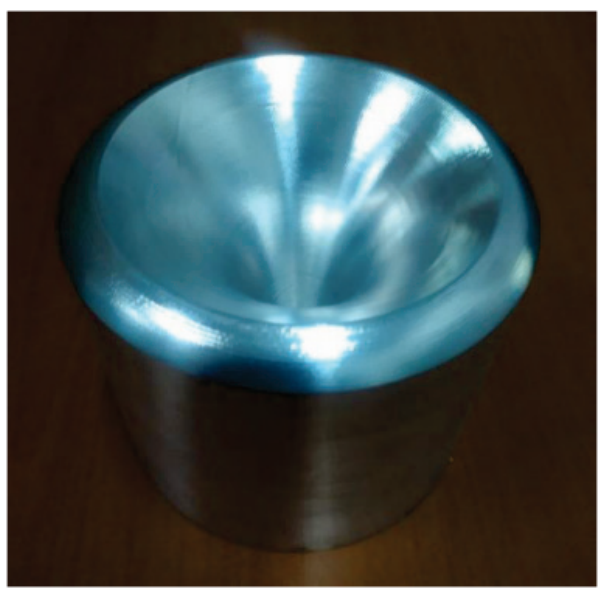

(a) The sample of HR

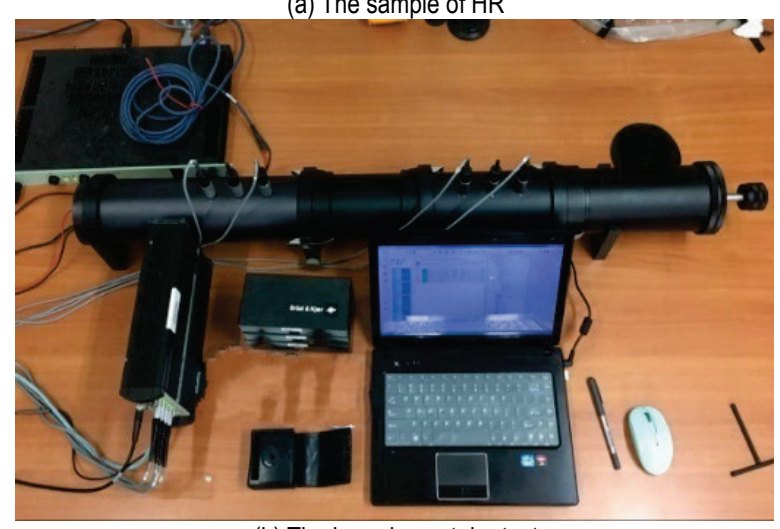

(b) The impedance tube test

Figure 3 The sample of HR and the equipment of sound absorption coefficient text

In order to describe the sound absorption coefficient and the resonance frequency of the resonator quantitatively, the sound absorption performance of the resonator sample was tested by the Brüel \& Kjær 4206-T impedance tube according to the ISO 10534-2 [13] standard established by the International Organization for Standardization. As shown in Fig. 3b, the sound source, which makes a plane acoustic wave having a frequency range of $50-180 \mathrm{~Hz}$, is located at the left end of the impedance tube, while the HR with an exponential neck is placed at the other side of the impedance tube. The double microphone method has been used to measure the response of sound. The two microphones are respectively located at the inner wall of the impedance tube at point 1 and point 2 , the distance between the two measuring points is $50 \mathrm{~mm}$, and the distance between the measuring point 1 and the plane of the resonator opening is $200 \mathrm{~mm}$. Thus, the sound pressure response at the two measuring points is processed by the transfer function method, and the sound absorption coefficient of the resonator can be calculated.

\subsection{The Noise Reduction Experiment of Cylindrical Shell with HR}

A noise reduction test platform for aluminum alloy reinforced cylindrical shell has been built at our previous work [14]. The experiment result showed that there were many peak noises in the response of the acoustic cavity in the cylindrical shell under the action of the reverberation sound source whose external loads are $117 \mathrm{~dB}$. For example, the noise peak $(83 \mathrm{~dB})$ at the first-order axial resonance frequency $(154 \mathrm{~Hz})$ of the acoustic cavity in the cylinder is still very high. So, the resonator has been applied to reduce the noise peak in the cylinder for the reason that it has a powerful sound absorption capability at its resonant frequency.

To reduce the noise peak in the cylinder cavity, the HR in Fig. 3a has been installed in the upper position of the cylindrical cavity, as illustrated in Fig. 4 where the resonant frequency of the resonator is $154 \mathrm{~Hz}$, the height, diameter and thickness of the cylindrical shell are $1125 \mathrm{~mm}, 1050$ $\mathrm{mm}$ and $1.2 \mathrm{~mm}$, respectively.

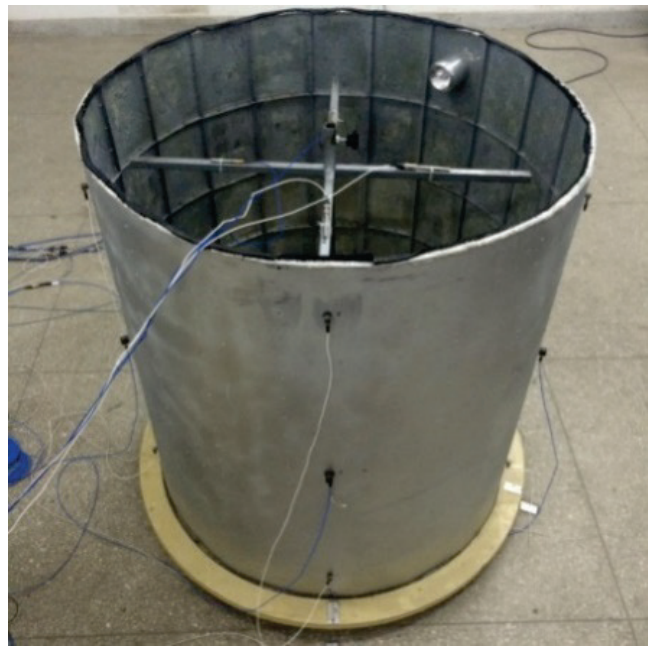

Figure 4 The Cylindrical Shell with HR

In the experiment, the resonator and the cylindrical shell are glued together, the upper and lower surfaces are sealed with a wooden cover, and the bottom is supported by a rubber block. The equipment used in the experiment 
contains C-MARK speaker (FT-04), the power amplifier (ME-8000), Equalizer (C-MARK A-907) and Acoustic sensor (PCB 130A23) where the speaker, power amplifier and Equalizer produce an external reverberant sound field whose overall sound pressure level is $117 \mathrm{~dB}$. The 24 acoustic sensors, which are distributed evenly in the inner cavity of the cylindrical shell, are used to measure the internal acoustic cavity response at the same time. Finally, the Prosig DATS 7.0 data analysis software is used to process the collected data.

\section{SIMULATION \\ 4.1 The Simulation of HR}

The material of HR which has an exponential neck is aluminum. According to the structural dimensions of the Helmholtz in Fig. 3a, the finite element model of the HR is established in the acoustic simulation software LMS Virtual.Lab, as shown in Fig. 5. The mesh of the HR divides these models more than 10000 triangular elements. The material properties of the HR are that Young's Modulus is $71 \mathrm{GPa}$, density is $2700 \mathrm{~kg} / \mathrm{m}^{3}$, Poisson's ratio is 0.34 . The mesh of the fluid inside and outside the resonator divides the tetrahedral element. The material property is air density is $1.2122 \mathrm{~kg} / \mathrm{m}^{3}$, the speed of sound in the air is $340 \mathrm{~m} / \mathrm{s}$.

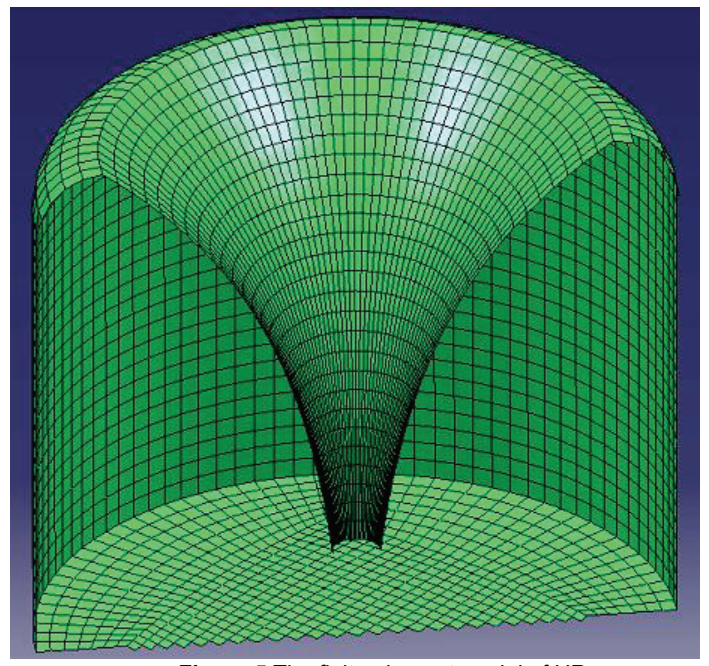

Figure $\mathbf{5}$ The finite element model of HR

The impedance coefficient of the resonator is quantitatively described by the impedance tube method. In LMS Virtual.Lab, the virtual impedance tube method simulation model is established, as shown in Fig. 6 The finite element model of the resonator is placed at the end of the impedance tube and the acoustic excitation is applied at the sound source end. Where, $x_{1}$ is the distance of the reference point 1 and the $\mathrm{HR}, \mathbf{s}$ is the distance between the two reference points. Thus, two responses of the reference point on the impedance tube can be obtained. The length of the impedance tube is set as 1 meter to avoid the first-order axial frequency of the air in the impedance tube which might be too low coupling with the resonant frequency of the resonator. It is assumed that all acoustic envelope surfaces of the resonator and the impedance tube are rigid, and the modal damping is 5\% [16]. The acoustic excitation of the sound source uses velocity boundary conditions. The real and imaginary parts of the sound pressure at the reference point 1 and point 2 can be obtained by the acoustic-vibration coupling calculation, and the sound absorption coefficient of the HR can be obtained [17].

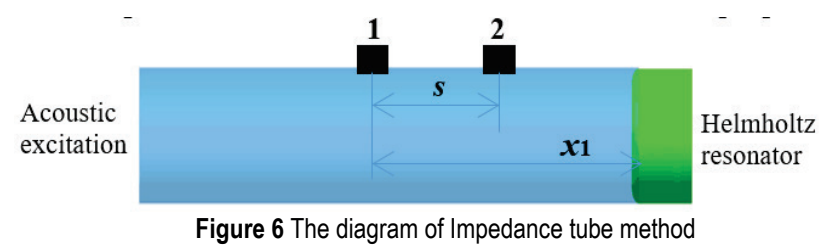

The experimental results of the HR sound absorption coefficient and the simulation results are shown in Fig. 7. At the resonant frequency $(154 \mathrm{~Hz})$, the sound absorption coefficient of the resonator is 0.92 , which is very close to the experimental results. Therefore, the finite element model of HR and the simulation method are reliable, and the simulation methods can reflect the actual sound absorption effect of the resonator.

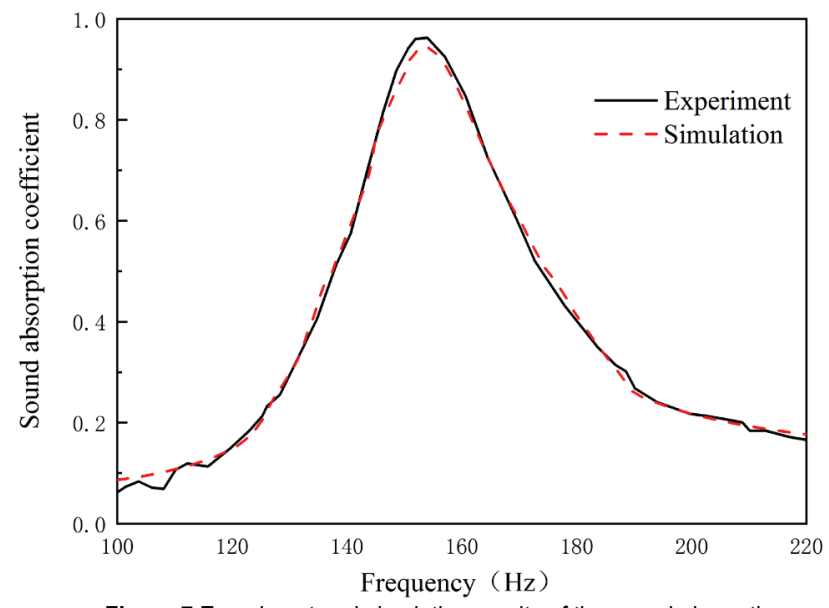

Figure 7 Experiment and simulation results of the sound absorption coefficient

\subsection{Acoustic Simulation of Cylindrical Shell with HR}

As shown in Fig. 8, the three-dimensional FEM simulation model of a cylindrical shell with a resonator is built by using the commercial software (Hypermesh). The cylindrical shell and the upper and lower covers are meshed by the CQUAD tetrahedral unit, while the inner and outer acoustic chambers of the cylindrical are meshed by the CHEXA hexahedral unit. The cylindrical shell and the resonator are meshed by the CHEXA unit nodes to achieve consolidation. Then, the finite element model was imported into the acoustic simulation software Virtual.Lab for pre-processing to define the structure, fluid, and material properties. Where the cylindrical shell and rib material are aluminum, its density is $2700 \mathrm{~kg} / \mathrm{m}^{3}$, Young's modulus is $70 \mathrm{GPa}$ and Poisson's ratio is 0.33 . The upper and lower wooden cover is considered to be isotropic materials, and its density is $800 \mathrm{~kg} / \mathrm{m}^{3}$, Young's modulus is $2 \mathrm{GPa}$, Poisson's ratio is 0.4 . The HR parameters are described in Section 4.1. The incident wave and the reflected wave outside the cylindrical shell are defined as an automatic matching layer, and the connection between the wooden cover and the cylindrical shell is defined as coupling. 


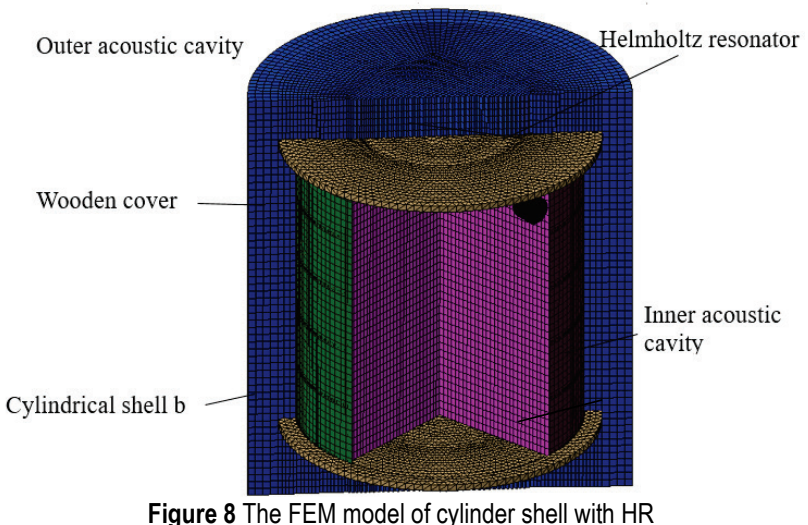

In the simulation, the equivalent reverberation source is replaced by a uniformly distributed 24 column plane waves which surround the cylindrical shell, and the external load was imported by the actual measurement results of the reverberation chamber in the experiment. Then, the average response of 1000 measurement points in the cylindrical shell is obtained by the coupling solution calculation. The step size of the calculation is set to $1 \mathrm{~Hz}$. The inner acoustic response of cylindrical shell with HR at the frequency of $130-180 \mathrm{~Hz}$ is obtained. The experiment and simulation calculation results are shown in Fig. 9.

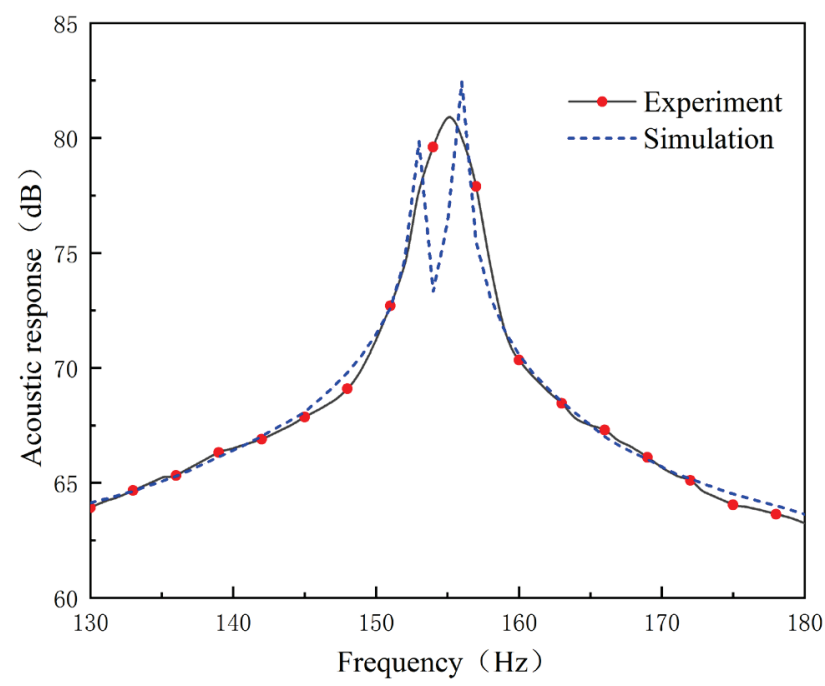

Figure 9 The inner cavity response of the experimental and simulation

The results of simulation and experiment of the cylindrical shell with HR have the same trend, and the effective sound absorption frequency range is $149-159 \mathrm{~Hz}$. There is no new response peak around the original normal frequency in the experiment results, while the simulation results show that the response of the cylinder at the resonant frequency is significantly reduced and two new noise peaks are founded around the resonant frequency of the HR. The experiment result is the average of 24 points in the cavity, while the simulation result is the average of 1000 points in the cavity. Thus, the newly formed response peaks are not captured in the experiment. Therefore, the experimental results only reflect the changes at the resonance frequency of the resonator, and the simulation results more accurately reflect the sound field in the cylindrical cavity at the resonant frequency of the resonator.

\section{DISCUSSION}

The air in the cylindrical cavity can be regarded as a closed cavity in the study of noise reduction in the cylindrical cavity. When the resonance frequency of the $\mathrm{HR}$ is consistent with the modal frequency of the closed cavity, the HR can effectively reduce the amplitude of the sound pressure at the modal frequency. The space occupied by a single HR is less than $1 \%$ of the volume of the entire cylindrical shell cavity. Therefore, the installation location and the number of HR need to be chosen to achieve the best noise reduction effect.

\subsection{The Installed Location of HR}

As shown in Fig. 10, the HR's are placed in three different positions in the cylindrical cavity for the reason that the first-order axial mode of the acoustic cavity in the cylindrical shell is extended axially and the first order mode has different relative amplitudes at different positions. Under the excitation of the reverberation source, the sound pressure distribution at different installation positions in the cylindrical cavity is obtained by simulation.

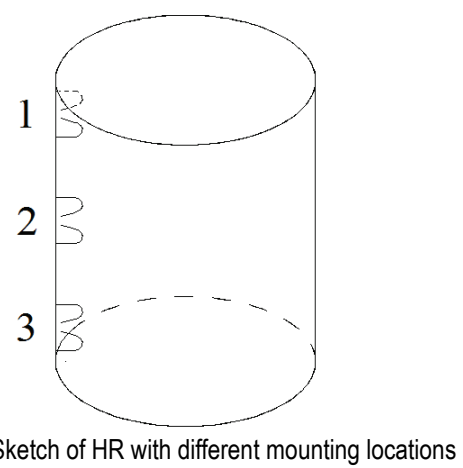

Fig. 11 shows the inner cavity noise response between different install positions. At the frequency range of 130$180 \mathrm{~Hz}$, the sound pressure response of 1000 points in a cylindrical cavity is calculated under the external sound field reverberation excitation. The relationship between the average sound pressure of 1000 points in the cylindrical cavity and frequency can be obtained, as shown in Fig. 11. Then, the response of the cylindrical cavity at the frequency range $130-180 \mathrm{~Hz}$ is analyzed.

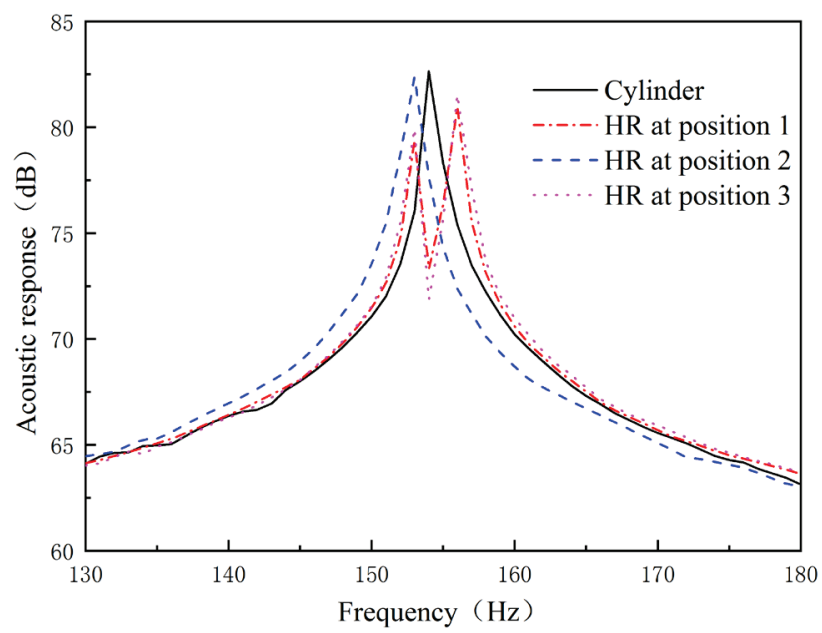

Figure 11 The acoustic response at the different installed positions of HR 
From Fig. 11, when the HR was installed at the position 1 and position 3, the sound pressure level spectrum in the cylindrical cavity is basically the same, the sound pressure level of the first-order normal frequency of the acoustic cavity is significantly reduced. Although two new response peaks around the original normal frequency have emerged, the noise peak amplitude is reduced by more than $10 \mathrm{~dB}$ at a frequency of $154 \mathrm{~Hz}$ compared to the original response peak amplitude, and the average sound pressure level is reduced by $2.87 \mathrm{~dB}$. When the HR was installed at position 2, the response peak amplitude of the inner acoustic cavity compared to no installation is almost unchanged, but the normal frequency is changed. The total noise reduction of the resonator installed at positions 1 is $1.15 \mathrm{~dB}$, position 2 is $0.42 \mathrm{~dB}$, and position 3 is $1.04 \mathrm{~dB}$, respectively.

\subsection{The Installation Number of HR}

From the previous study, it is obvious that when a single HR is installed in the cylindrical cavity, two new response peaks will be formed near the original resonance frequency. The frequency range of HR can be increased and the sound absorption performance can be improved by increasing the number of resonators [16]. The cylindrical shell structure is an axisymmetric structure. The different number of HR with the same resonance frequency is installed symmetrically in the cylindrical shell at position 1 shown in Fig. 10. The finite element model is established respectively and the cylindrical cavity response is calculated at the frequency range of 130-180 Hz. The results of the simulation are shown in Fig. 12. The number in front of HR indicates the number of HR installed on the cylinder.

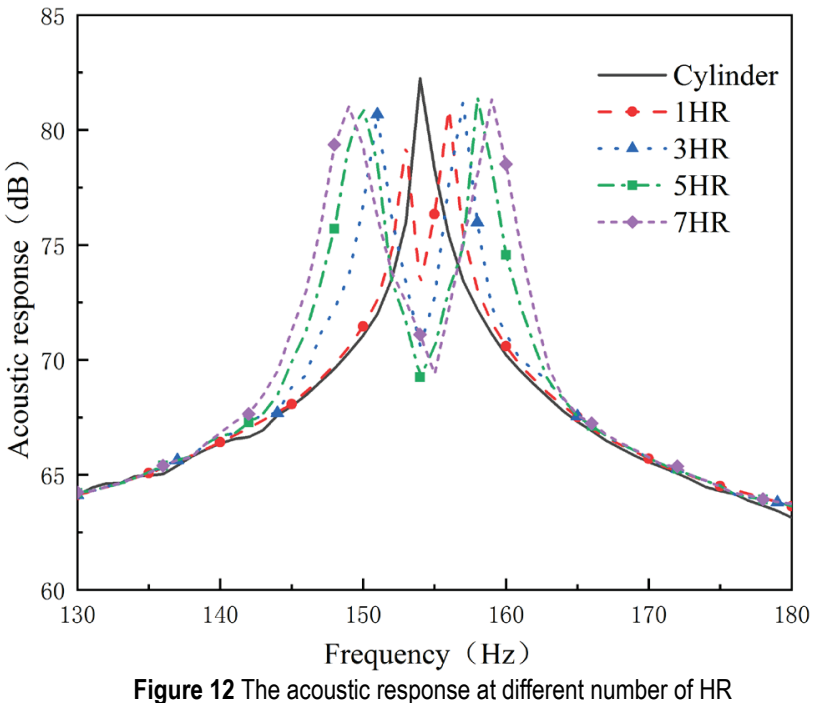

At the frequency range of $145-165 \mathrm{~Hz}$, the response peak at the first-order normal frequency of $154.0 \mathrm{~Hz}$ can be reduced greatly by installing different number of HR's, and two new noise peaks are founded around the resonant frequency. The distance between the two new response peaks is increased with the number of the HR's installed, which indicates that the frequency ranges of noise reduction in the cylindrical cavity gradually increased with the number of the HR's installed. The noise reduction of the cylindrical cavity which installed the different number of HR's is $0.92 \mathrm{~dB}, 1.13 \mathrm{~dB}, 0.09 \mathrm{~dB}$ and $0.98 \mathrm{~dB}$. It is known that the amount of noise reduction increases to the maximum first, and then oscillates below the maximum value. That is, the optimal installation number of the resonant frequency resonator in the cavity is three.

\subsection{The Resonant Frequencies of the HR}

The installation of a single resonant frequency resonator will form two new peak noise points in the resonance frequency accessory [18]. When multiple HR's with the same response frequency are installed in the cylindrical cavity, there is no significant increase in the sound absorption frequency range of the cylindrical cavity. Therefore, the HR which has the same resonant frequency as the frequency at the newly formed noise peak point is installed in the cylindrical shell, and the noise reduction effect of the resonators which have the same resonance frequency and different resonance frequency are compared under the condition of the same number HR. The cavity response of the cylindrical shell under different working conditions was simulated, as shown in Fig. 13. The number in front of HR indicates the number of HR's, and the number in front of RF (resonant frequency) indicates the number of resonant frequency installed on the cylinder.
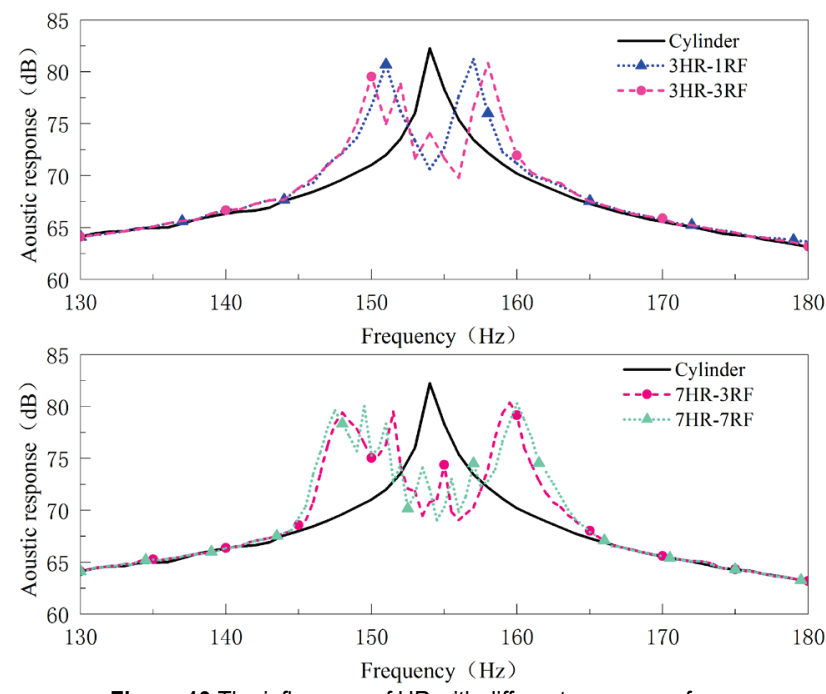

Figure 13 The influences of HR with different resonance frequency

The effective sound absorption frequency ranges of the HR's which have the same frequency are consistent with the effective sound absorption frequency ranges of the HR's which have a different frequency at the same number of HR. When the number of installed HR's is 3, the noise reduction in the cavity of the same resonant frequency and three different resonant frequencies is $1.13 \mathrm{~dB}$ and $1.32 \mathrm{~dB}$. When the number of installed HR's is 7, the noise reduction in the cavity of three different resonant frequencies and seven different resonant frequencies is $1.76 \mathrm{~dB}$ and $1.81 \mathrm{~dB}$ respectively. Therefore, when the number of resonators installed in the cylindrical cavity is the same, the noise reduction effect of different resonance frequency resonators is better than that of the same resonance frequency resonators at the condition of installing the same number HR. 


\section{CONCLUSION}

In this paper, the resonance frequency and sound absorption coefficient of HR's with three kinds of neck types are studied by using the lumped parameter method. The simulation model of the cylindrical shell with HR is established and calculated by the finite element method (FEA). The influences of the installation position, the number of installations and the resonance frequency of the resonators in the cylindrical cavity on the noise reduction effect in the cylindrical cavity are discussed. The research results show that

(1) If the shape and the volume of the resonators are similar, the HR of the exponential neck has a larger effective length and a lower resonance frequency than the other types of HR. Respectively, the HR with an exponential section opening has a smaller volume in the conditions of their resonance frequency are the same.

(2) When the resonator is installed at the position 1 and 3 , the noise peak in the original cavity drops by more than $10 \mathrm{~dB}$. Therefore, in order to achieve the best effect of noise reduction, the installed position of HR should be located at the place where the cylinder cavity mode amplitude is largest.

(3) Increasing the number of resonators has a better effect on the inner cavity noise reduction, and the optimal number of resonators is 3 , while the noise reduction frequency range can be widened by increasing the number of resonators with a different resonant frequency.

\section{Acknowledgments}

This work is supported by Fundamental Research Funds for the Central Universities (WUT: 2017IB016). This work was finished at Wuhan University of Technology (WUT), Wuhan.

\section{REFERENCES}

[1] Balakrishnan, P. \& Srinivasan, K. (2019). Impinging jet noise reduction using non-circular jets. Applied Acoustics, 143, 19-30. https://doi.org/10.1016/j.apacoust.2018.08.016

[2] Rempel, D., Antonucci, A., Barr, A., et al. (2019). Pneumatic rock drill vs. electric rotary hammer drill: Productivity, vibration, dust, and noise when drilling into concrete. Applied Ergonomics, 74, 31-6. https://doi.org/10.1016/j.apergo.2018.08.005

[3] Tsutsumi, S., Takaki, R., Takama, Y., et al. (2012). Hybrid LES/RANSsimulations of transonic flowfield around a rocket fairing. 30 ${ }^{\text {th }}$ AIAA Applied Aerodynamics Conference, New Orleans, USA. https://doi.org/10.2514/6.2012-2900

[4] Yuan, C., N. Bert, R., Otto, B., et al. (2013). Experimentalnumerical study and optimization of sound insulation of a finite composite cylinder. Engineering Analysis with Boundary Elements, 37(2), 250-259. https://doi.org/10.1016/j.enganabound.2012.09.013

[5] Arianespace. Ariane 5 user's manual (2011). Issue 5, Rev. 1. Evry, Arianespace.

[6] Mitsubishi Heavy Industries. H-IIA user's manual (2015). Version 4.0[M]. Tokyo: Mitsubishi Heavy Industries.

[7] Tang, S. K. (2005). On Helmholtz resonators with tapered necks. Journal of sound and vibration, 279, 1085-1096. https://doi.org/10.1016/j.jsv.2003.11.032

[8] Liu, H., Zheng, S., Lian, X., et al. (2014). The acoustic performance prediction of Helmholtz resonator with a conical neck. Acta Acustica, 39(3), 353-359.
[9] Borchers, I. U., Laemmlein, S. T., Bartels, P., et al. (1997). Acoustic protection on payload fairings of expendable launch vehicles. United States: 5670758. 1997-09-23.

[10] Cai, C. Z. \& Mak, C. M. (2018). Noise attenuation capacity of a Helmholtz resonator. Advances in Engineering Software, 116, 60-6. https://doi.org/10.1016/j.advengsoft.2017.12.003

[11] Dunn, F., Hartmann, W. M., Campbell, D. M., et al. (2015). Springer handbook of acoustics. Springer.

[12] Komkin, A. I., Mironov, M. A., \& Bykov, A. I. (2017). Sound Absorption by a Helmholtz Resonator. Acoustical Physics, 63, 385-392. https://doi.org/10.1134/S1063771017030071

[13] European Standard EN ISO 10534-2:2001 (2001). Determination of sound absorption coefficient and impedance tubes, transfer function method.

[14] Li, B., Li, J., Yan, S., et al. (2017). Experiment and Simulation Analysis on Noise Attenuation of $\mathrm{Al} / \mathrm{MF}$ Cylindrical Shells. Shock and Vibration, (1), 1-8. https://doi.org/10.1155/2017/6980501

[15] Bellucci, V., Flohr, P., Paschereit, C. O., et al. (2004). On the use of Helmholtz resonators for damping acoustic pulsations in industrial gas turbines. Journal of engineering for gas turbines and power, 126(2), 271-275. https://doi.org/10.1115/1.1473152

[16] Utsuno, H., Tanaka, T., Fujikawa, T., et al. (1989) Transfer function method for measuring characteristic impedance and propagation constant of porous materials. The Journal of the Acoustical Society of America, 86(2), 637-643. https://doi.org/10.1121/1.398241

[17] Cai, C. \& Mak, C. M. (2018). Hybrid noise control in a duct using a periodic dual Helmholtz resonator array. Applied Acoustics, 134, 119-24. https://doi.org/10.1016/j.apacoust.2018.01.015

[18] Xu, M. B., Selamet, A., \& Kim, H. (2010). Dual Helmholtz resonator. Applied Acoustics, 71(9): 822-829. https://doi.org/10.1016/j.apacoust.2010.04.007

\section{Contact information:}

\section{Wenjie YAN}

1) Hubei Key Laboratory of Theory and Application of Advanced Materials Mechanics, Wuhan University of Technology, Wuhan, Hubei 430070, China 2) School of Mechanical Engineering, Henan Mechanical and Electrical Vocational College, Xinzheng, Henan 451191, China

\section{Shilin YAN}

(Corresponding author)

Hubei Key Laboratory of Theory and Application of Advanced Materials

Mechanics, Wuhan University of Technology,

122 Luoshi Road,Wuhan, Hubei 430070, China

E-mail: yanshilin408@yeah.net

Bin LI

School of Mechanical Engineering, Wuhan Polytechnic University, Wuhan, Hubei 430023, China

\section{Yongjing LI}

Hubei Key Laboratory of Theory and Application of Advanced Materials

Mechanics, Wuhan University of Technology,

Wuhan, Hubei 430070, China 